\title{
Performance Comparison of Optimum and MMSE Receivers With Imperfect Channel Estimation for VSF-OFCDM Systems
}

\author{
Bin Xia, Jiangzhou Wang, Senior Member, IEEE, and Mamoru Sawahashi, Member, IEEE
}

\begin{abstract}
Performances of optimum and minimum mean square error (MMSE) receivers for variable spreading factor orthogonal frequency and code division multiplexing (VSFOFCDM) systems are compared in this paper. In VSF-OFCDM systems, the existence of multicode interference (MCI) in the frequency domain due to frequency-selective fading channels dramatically degrades the system performance. A quasi-analytic bit error rate performance is presented in the presence of imperfect channel estimations. Numerical results show that with linear computation complexity the MMSE receiver can improve system performance significantly by suppressing the MCI,although it cannot perform as well as the optimum receiver. Thus, with a small number of code channels, an optimum receiver can be employed to achieve better performance, whereas the MMSEreceiver is suitable for a system with a large number of code channels due to simple complexity. In addition, the MMSE receiver is more robust than the optimum receiver to the different configurations of system parameters, e.g., spreading factors. Moreover, it is found that pilot channel power should be carefully assigned by making tradeoffs between the channel estimation quality and the received SNR for each code data channel.
\end{abstract}

Index Terms - Channel estimation, fading channels, minimum mean square error (MMSE), orthogonal frequency division multiplexing (OFDM), optimum receiver, time and frequency domain spreading.

\section{INTRODUCTION}

$\mathbf{R}$ ECENTLY, the orthogonal frequency and code division multiplexing (OFCDM) system has been proposed in [1] for future high-speed wireless transmission. In OFCDM systems, each code channel is spread in the frequency domain over a number of subcarriers by a dedicated spreading code so that it can make use of frequency diversity over subcarriers. Another advantage of OFCDM is that its symbol duration is much larger than the channel delay spread so that it mitigates the multipath effect, i.e., intersymbol interference (ISI). Therefore, OFCDM is a promising candidate for future broadband wireless communications. In addition to frequency domain spreading,

Manuscript received April 1, 2004; revised June 30, 2004; accepted November 8,2004 . The editor coordinating the review of this paper and approving it for publication is $\mathrm{C}$. Xiao.

B. Xia was with the Department of Electrical and Electronic Engineering, University of Hong Kong, Hong Kong. He is now with the Alcatel Shanghai Bell Company, Ltd., Shanghai, China.

J. Wang is with the Department of Electrical and Electronic Engineering, University of Hong Kong, Hong Kong (e-mail: jwang@eee.hku.hk).

M. Sawahashi is with the Internet Protocol Radio Network Development Department, NTT DoCoMo Inc., Kanagawa 239-8536, Japan.

Digital Object Identifier 10.1109/TWC.2005.857998 variable spreading factor (VSF) OFCDM has been proposed in [2], [7], which employs time domain spreading as well, but with higher priority than frequency domain spreading for flexible deployment in different channel environments. Due to the orthogonality of time domain spreading codes, there is no multicode interference (MCI) in the time domain when code channels are orthogonal in slow fading or additive white Gaussian noise (AWGN) channels.

However, the received OFCDM signal contains MCI due to the loss of orthogonality among code channels suffering from different fading on different subcarriers. Although spreading over more subcarriers gains higher frequency diversity, more MCI is caused accordingly. Therefore, in VSF-OFCDM systems, spreading factors for time and frequency domain spreading should be carefully designed to maximize the overall received signal quality, which mainly depends on the tradeoff between frequency diversity and MCI effects. As these factors are much related with channel load and other cell interference, it is necessary to provide an analytical method to determine spreading factors according to various channel conditions.

So far, minimum mean square error (MMSE) has proved to be effective to suppress cochannel interference, e.g., multiuser interference, and thereby provides better performance. Until now, some simulation results have been presented for OFCDM systems [1], [2], or code division multiple access (CDMA) systems [5]. Some analytical results for MMSE in CDMA systems, which are based on Gaussian approximation of multiuser interference, have been presented in [3] and [4]. The assumption works well in the case of a large number of users in CDMA systems. However, in VSF-OFCDM systems, since code channels with different time domain spread code contribute little MCI, MCI cannot be approximated as Gaussian when the number of code channels contributing MCI is small, which is far smaller than the number of code channels. Although Grant and Cavers [6] studied the effect of imperfect channel estimation for the MMSE receiver in the case of a multitransmitter system, it is also based on a Gaussian assumption and cannot be applied to VSF-OFCDM systems directly.

In this paper, we provide a quasi-analytical bit error rate (BER) performance for the MMSE detector in VSF-OFCDM systems with imperfect channel and noise power estimation. Moreover, an analytical BER performance for optimum detector is also provided for comparison.

This paper is organized as follows. In Section II, the transmit model and scheme for the assignment of spreading codes are 


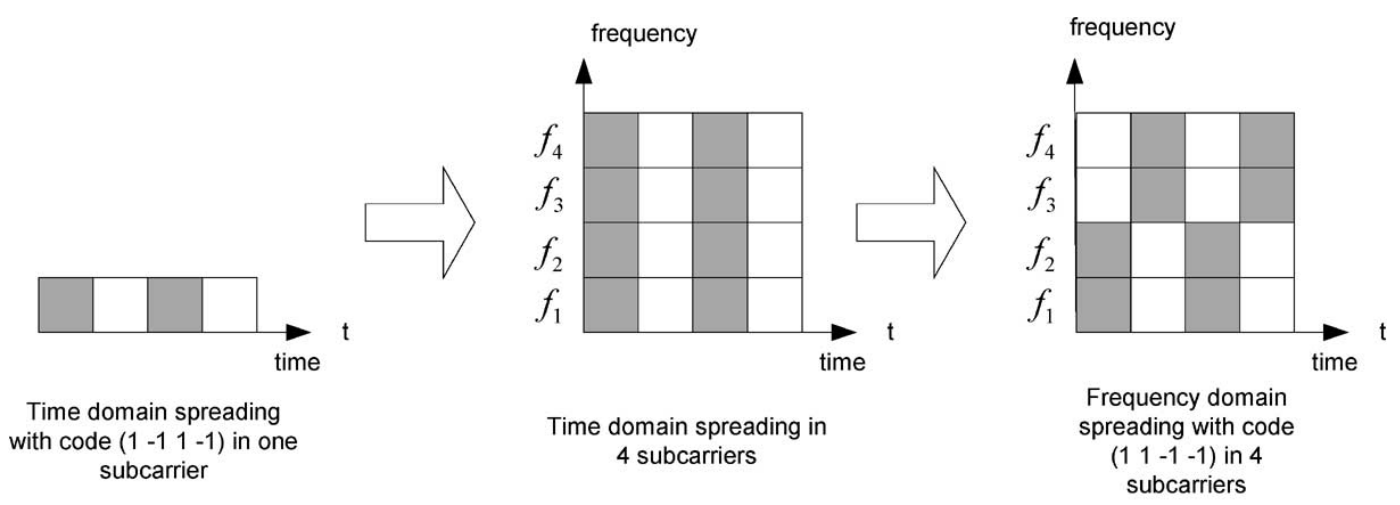

stands for 1, $\square$ stands for -1 )

Fig. 1. Example for 2-D spreading with $N=N_{T} \times N_{F}=4 \times 4$.

presented. Then, two receiver structures (optimum detector and MMSE detector) are described in detail, including pilot-aided channel and noise power estimation algorithms. In Section III, we derive analytical expressions for optimum and MMSE detectors, respectively. In Section IV, comparison and discussion on numerical BER results with variable parameters for different detectors are presented. Finally, some conclusions are drawn in Section V.

The following notations are used throughout the paper. Symbols with boldface type represent matrices or vectors, and the superscripts $T$, *, and $H$ stand for transpose, conjugate, and conjugate transpose, respectively.

\section{SYSTEM DESCRIPTION}

\section{A. Transmitter Model}

In VSF-OFCDM systems, for each code channel with a spreading factor of $N=N_{T} \times N_{F}$, the transmitter performs two-dimensional (2-D) spreading by using a time domain spreading code with length (or spreading factor) of $N_{T}$ and a frequency domain spreading code with length (or spreading factor) of $N_{F}$. Both time and frequency domain spreading codes are generated from orthogonal VSF (OVSF) codes. Spreading factors for time and frequency domain spreading are varied according to channel conditions to achieve high system performance. Fig. 1 shows an example for 2-D spreading. Since channels with different time domain spreading codes are orthogonal to each other, the MCI from code channels with different time domain spreading codes approaches zero in slow fading and AWGN channels. Frequency diversity is provided due to frequency domain spreading. Similarly, frequency domain spreading codes are orthogonal to each other. In Gaussian channels, there is no MCI among frequency domain spreading codes. However, because fading parameters on subcarriers bearing the same information are not the same, orthogonality in the frequency domain no longer maintains among code channels at the receiver. Thus, MCI is caused.

The transmitter block diagram for the forward link of a single-cell OFCDM system is shown in Fig. 2. Consider the $k$ th data stream. The symbol sequence is first serial-to-parallel converted to $M / N_{F}$ (suppose $M / N_{F}$ is an integer and $M$ is the total number of subcarriers) parallel sequences and then spread by a time domain spreading code $c_{T, k}^{(\mathrm{CH})}$. Each time domain spreading signal is duplicated into $N_{F}$ parallel copies for $N_{F}$ subcarriers. Each copy is multiplied by a chip of the frequency domain spreading code, which is the combination of a short channelization code $c_{F, k}^{(C H)}(n)$ and a cell-specific long-scrambling code $c^{(S C)}(n) . c_{F, k}^{(C H)}$ and $c_{T, k}^{(C H)}$ are realvalued binary channelization codes taking the value of \pm 1 , whereas $c^{(S C)}$ is a real-valued binary scrambling code that is the same for all code channels in a cell. $K$ is the number of parallel channels code multiplexed with different combinations of time and frequency domain channelization codes $\left\{c_{T, k}^{(C H)}, c_{F, k}^{(C H)}, k=1, \ldots, K\right\}$. Therefore, in VSF-OFCDM, each data symbol is impressed over $N_{F}$ subcarriers by $N_{T}$ OFCDM symbols (chips) in each subcarrier. To aid channel estimation at the receiver, a common pilot channel with spreading factor of $N_{\text {Pilot }}$ is employed and code multiplexed over each subcarrier. Note that $E_{D}$ is the chip energy of the transmitted symbol on data channel, $\beta$ is the power ratio of pilot channel to one code data channel, and for the $m$ th $(m=1, \ldots, M)$ subcarrier the pilot symbol $d_{m, P}$ is known to the receiver and the spreading code for pilot channel is an all-1 sequence. In order to exploit frequency diversity, a frequency interleaver is employed before orthogonal frequency division multiplexing (OFDM) modulation. Therefore, the largest possible frequency separation between subcarriers carrying the same information is achieved. For example, subcarriers $\left\{1,2, \ldots, N_{F}\right\}$ are bearing the same information; however, they are separated as much as possible in spectrum to achieve maximum frequency diversity gain. After frequency interleaving with inverse fast Fourier transform (IFFT), spread signals occupy all $M$ subcarriers. Similar to OFDM, in the transmitter, a guard interval is used between every OFCDM symbol to avoid the ISI caused by multipath propagation.

In order to meet the orthogonality property of different codes, it is desired to assign code channels with different time domain spreading codes and the same frequency domain spreading code. Similar to the example in [2], a pilot channel is assumed with a spreading factor of $N_{\text {Pilot }}=16 \times 1$ and $K$ code channels are assumed with a spreading factor of $4 \times N_{F}$, where $N_{T}=4$. The pilot channel is assigned 


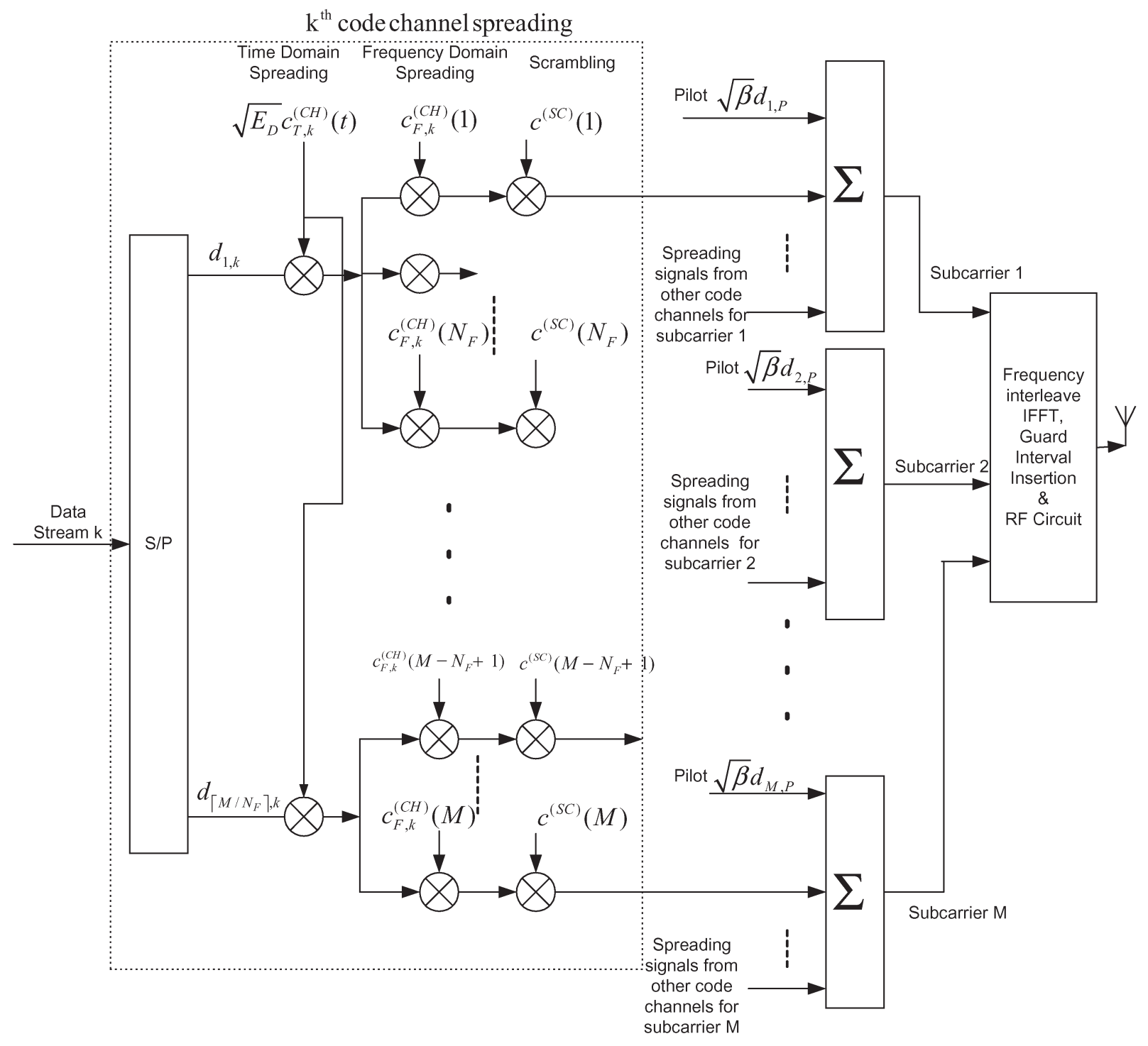

Fig. 2. Transmitter diagram for the forward link of the VSF-OFCDM system.

with the code $C_{16,1}$ in the OVSF code tree shown in Fig. 3. To maintain orthogonality between code channels, all mother codes of $C_{16,1}$ cannot be used. Thus, when $K \leq 3$, the $K$ code channels are assigned with different time spreading codes from the set $\left\{C_{4,2}, C_{4,3}, C_{4,4}\right\}$ and the same frequency domain spreading code $C_{N_{F}, 1}$. Note that codes in $\left\{C_{4,2}, C_{4,3}, C_{4,4}\right\}$ are orthogonal. However, when $K>3$, some code channels will be assigned with the same time domain spreading code from $\left\{C_{4,2}, C_{4,3}, C_{4,4}\right\}$, and distinguished by assigned different frequency domain spreading codes. In general, when $K<N_{T}$, the $K$ code channels can be assigned with different time domain spreading codes, but the same frequency domain spreading code $C_{N_{F}, 1}$, so that $\mathrm{MCI}$ in time domain is avoided. Although there are $N_{F}$ different frequency domain spreading codes available, only $N_{T}-1$ different time domain spreading codes are available, since one remained code $C_{N_{T}, 1}$ cannot be used due to its connection with the pilot channel. Thus, the maximum number of codes available is $\left(N_{T}-1\right) N_{F}$, which must be equal to or greater than $K$. When $K \geq N_{T}$, where $K$ is assumed to be integer times of $N_{T}-1$, the same $N_{T}-1$ codes have to be assigned repeatedly with the other different frequency domain spreading codes. Then, MCI may occur due to different fading gains on subcarriers. So, with this spreading code allocation strategy, for the $k$ th code channel, its spreading codes for frequency and time domain spreading can be derived from the tree in Fig. 3 as $C_{N_{F}, k_{f}}$ and $C_{N_{T}, \hat{k}}$, respectively, where $k_{f}=\left\lceil k /\left(N_{T}-1\right)\right\rceil \leq N_{F}$, $\hat{k}=k-\left(k_{f}-1\right)\left(N_{T}-1\right)+1$ and $\lceil x\rceil$ denotes the smallest integer no less than $x$. For the $k$ th code channel, it suffers from MCI only from other $K_{c}-1$ code channels, where $K_{c}=\left\lceil K /\left(N_{T}-1\right)\right\rceil \leq N_{F}$ is the number of code channels employing the same time domain spreading code $C_{N_{T}, \hat{k}}$. Referring to the original OFCDM system in [1], where no time domain spreading is employed, MCI is from all other $K-1$ code channels. However, in VSF-OFCDM systems, MCI is only from a small number $\left(K_{c}-1\right)$ of code channels. Thus, MCI is reduced significantly, but at the cost of a small frequency diversity for a given spreading factor. Therefore, time domain and frequency domain spreading factors should be optimized by considering both effects of MCI and frequency diversity. 


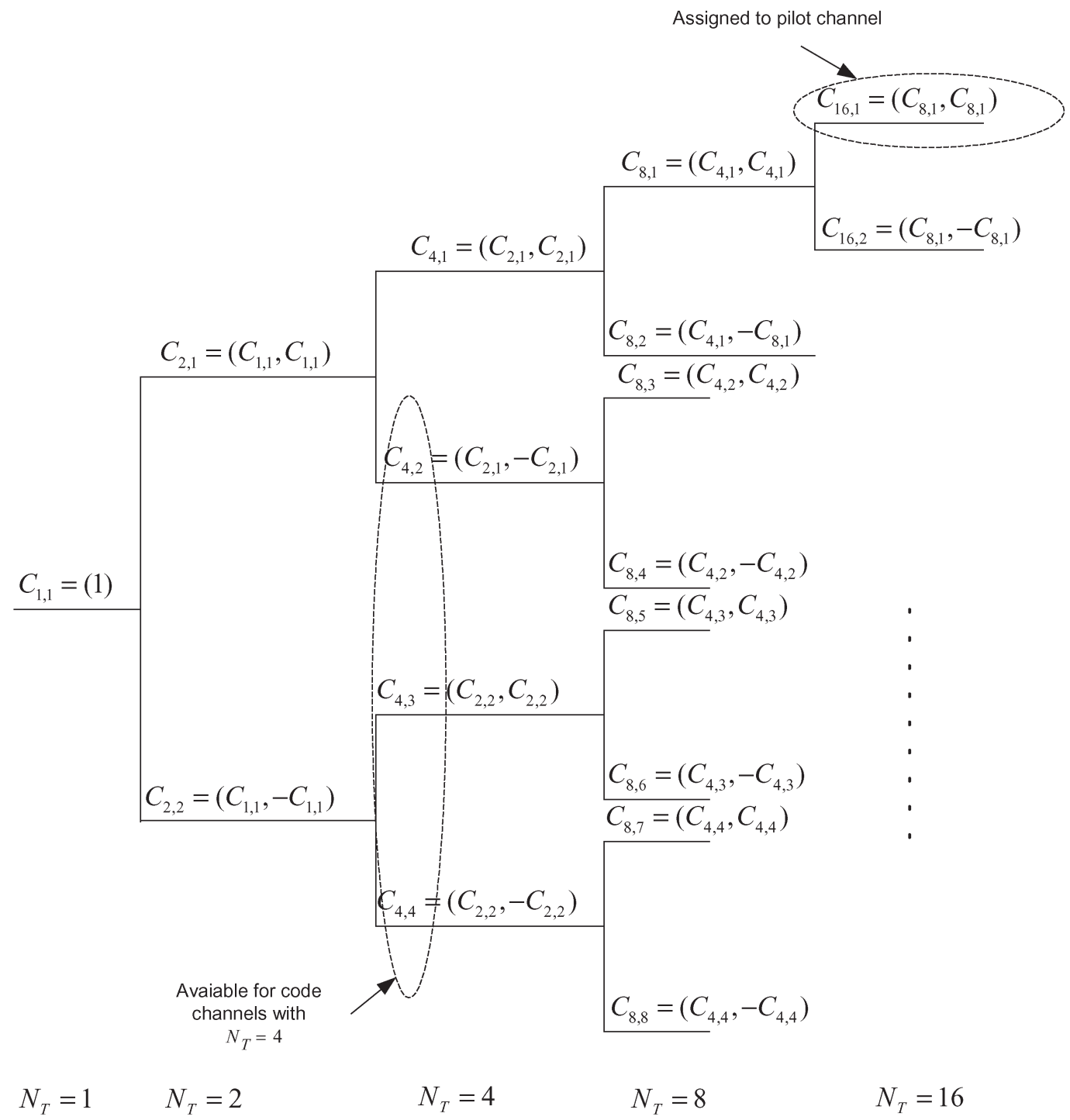

Fig. 3. Example for the assignment of time domain spreading codes for VSF-OFCDM system.

\section{B. Receiver Model}

At the receiver, as shown in Fig. 4, after guard interval deletion, FFT, and frequency deinterleaving, the equivalent received baseband signal on the $m$ th subcarrier for the $t$ th OFCDM symbol is given by

$$
\begin{aligned}
r_{m}(t)= & \sqrt{E_{D}} h_{m} c^{(S C)}(m) \sum_{k=1}^{K} c_{F, k}^{(C H)}(m) c_{T, k}^{(C H)}(t) \\
& \times d_{\left\lceil\frac{m}{N_{F}}\right\rceil, k}\left(t_{D}\right)+\sqrt{\beta E_{D}} h_{m} d_{m, P}+n_{m}(t) \\
= & \sqrt{E_{D}} h_{m} c^{(S C)}(m) \sum_{k=1}^{K} C_{N_{F}, k_{f}+1}(m) \\
& \times C_{N_{T}, \hat{k}}\left(t-\left(t_{D}-1\right) N_{T}\right) d_{\left\lceil\frac{m}{N_{F}}\right\rceil, k}\left(t_{D}\right) \\
& +\sqrt{\beta E_{D}} h_{m} d_{m, P}+n_{m}(t)
\end{aligned}
$$

where $h_{m}$ is the channel impulse response for the $m$ th subcarrier and $h_{m}$ is assumed to be an independent complex Gaussian random variable with zero mean and unit variance. Note that although fadings on adjacent subcarriers may be correlated to each other, since we are concerned about the received signal on subcarriers bearing the same information, which have been separated as far as possible in frequency domain, fading parameters on those subcarriers can be assumed independent of each other. $d_{\left\lceil m / N_{F}\right\rceil, k}\left(t_{D}\right)$ is the $t_{D}$ th transmitted symbol in the $k$ th data channel with $t_{D}=\left\lceil t / N_{T}\right\rceil . d_{m, P}$ is the pilot symbol for the $m$ th subcarrier and $n_{m}(t)$ is the zero mean AWGN noise with variance

$$
\sigma_{n}^{2}=\frac{1}{2} E\left\{n_{m}(t) n_{m^{\prime}}^{*}\left(t^{\prime}\right)\right\}=\sigma^{2} \delta\left(m-m^{\prime}\right) \delta\left(t-t^{\prime}\right)
$$

where $\delta\left(m-m^{\prime}\right)=1$ and 0 for $m=m^{\prime}$ and $m \neq m^{\prime}$, respectively.

Without loss of generality, data symbols $\left(d_{1, k}, k=\right.$ $1,2, \ldots, K)$ transmitted from the first subcarrier to the $N_{F}$ th subcarrier are assumed to be the desired symbols. After despreading the received signal (1) with different time domain spreading codes $C_{N_{T}, \hat{k}}$ for $\hat{k}=2,3, \ldots, N_{T}$, we can recover 


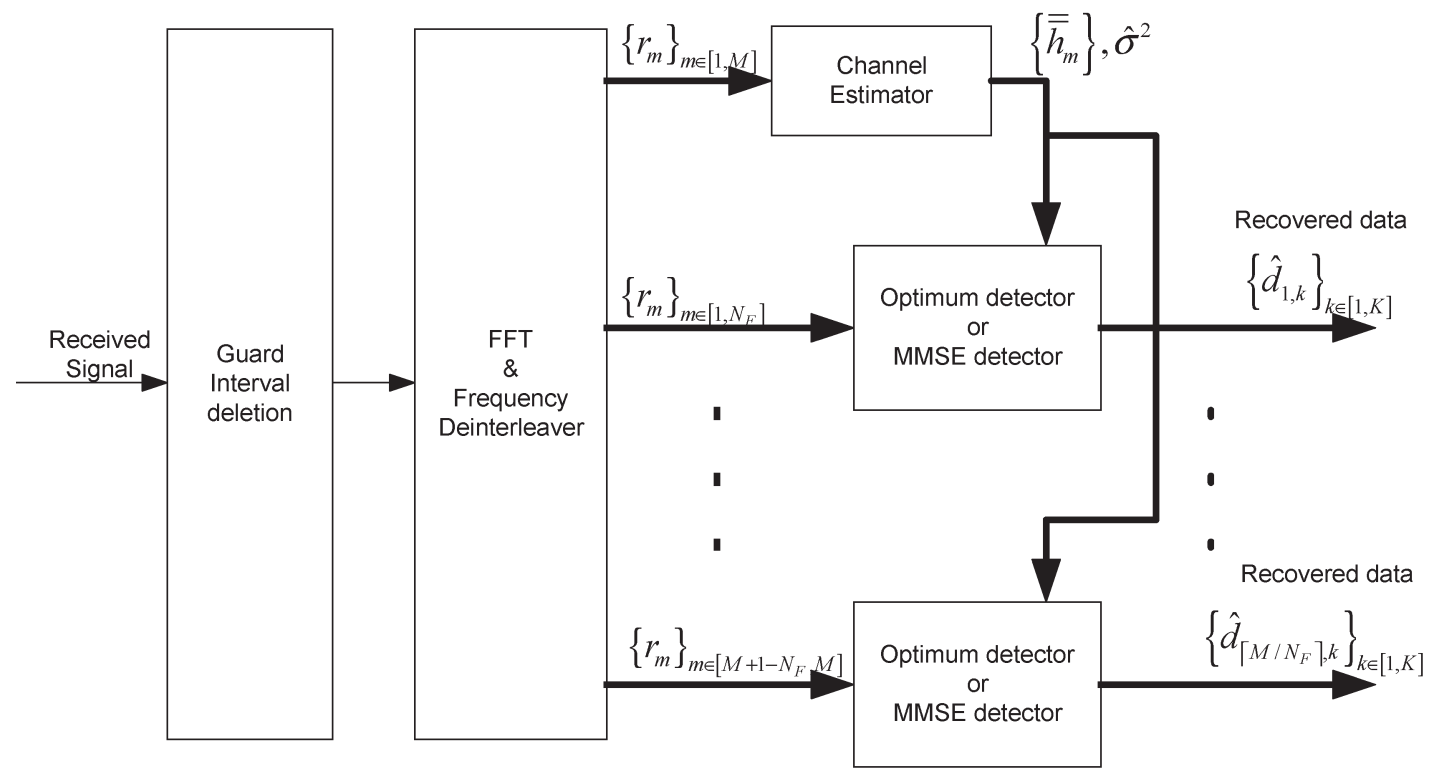

Fig. 4. Equivalent baseband receiver diagram for VSF-OFCDM.

information transmitted on all code channels by processing in the frequency domain. Assuming slow fading channels, the time domain despreading output on the $m$ th subcarrier is

$$
\begin{aligned}
y_{m, \hat{k}}\left(t_{D}\right)= & \sum_{t=\left(t_{D}-1\right) N_{T}+1}^{t_{D} N_{T}} r_{m}(t) C_{N_{T}, \hat{k}}\left(t-\left(t_{D}-1\right) N_{T}\right) \\
= & \sqrt{E_{D}} N_{T} h_{m} c^{(S C)}(m) \sum_{\hat{k}_{f}=1}^{K_{c}} C_{N_{F}, \hat{k}_{f}}(m) \\
& \times d_{1, \hat{k}-1+\left(\hat{k}_{f}-1\right)\left(N_{T}-1\right)}\left(t_{D}\right)+\eta_{m, \hat{k}}\left(t_{D}\right)
\end{aligned}
$$

where

$$
\eta_{m, \hat{k}}\left(t_{D}\right)=\sum_{t=\left(t_{D}-1\right) N_{T}+1}^{t_{D} N_{T}} n_{m}(t) C_{N_{T}, \hat{k}}\left(t-\left(t_{D}-1\right) N_{T}\right) .
$$

In (3), as code channels with different time domain spreading codes are orthogonal to each other, MCI only results from code channels with the same time domain spreading code and different frequency domain spreading codes. Then data symbols transmitted on code channels with time domain spreading code $C_{N_{T}, \hat{k}}$ can be recovered from (3). For convenience, the time domain despreading output is rewritten in vector form as

$$
\mathbf{Y}_{\hat{k}}\left(t_{D}\right)=\left[\begin{array}{llll}
y_{1, \hat{k}}\left(t_{D}\right), & y_{2, \hat{k}}\left(t_{D}\right), \ldots, y_{N_{F}, \hat{k}}\left(t_{D}\right)
\end{array}\right]^{T}
$$

In Fig. 4, channel estimator provides channel estimation $\left\{\overline{\bar{h}}_{m}\right\}$ and noise power estimation $\hat{\sigma}^{2}$. Using these estimations, optimum or MMSE detector is employed for symbol detection.

\section{Pilot-Aided Channel Estimator}

As channel and noise power estimations are required for both optimum and MMSE detectors, we first describe the pilot-aided channel estimator.

In slow fading channels, the output after time domain despreading for pilot channel on the $m$ th subcarrier can be expressed by

$$
\begin{aligned}
y_{m, 1}\left(t_{D}\right) & =\sum_{t=\left(t_{D}-1\right) N_{T}+1}^{t_{D} N_{T}} r_{m}(t) \\
& =\sqrt{\beta E_{D}} N_{T} h_{m} d_{m, P}+\sum_{t=\left(t_{D}-1\right) N_{T}+1}^{t_{D} N_{T}} n_{m}(t)
\end{aligned}
$$

where the second term is the AWGN component, which can be regarded as a Gaussian variable with zero mean and variance of $N_{T} \sigma^{2}$. Then we can get $E\left\{y_{m, 1}\left(t_{D}\right)\right\}=\sqrt{\beta E_{D}} N_{T} h_{m} d_{m, P}$. Thus, a simple channel estimation for the $m$ th subcarrier can be achieved by

$$
\begin{aligned}
\bar{h}_{m}\left(t_{D}\right) & =\frac{y_{m, 1}\left(t_{D}\right)}{\sqrt{\beta E_{D}} N_{T} d_{m, P}} \\
& =h_{m}+\sum_{t=\left(t_{D}-1\right) N_{T}+1}^{t_{D} N_{T}} \frac{n_{m}(t)}{\sqrt{\beta E_{D}} N_{T} d_{m, P}} \\
& =h_{m}+\Delta \bar{h}_{m}\left(t_{D}\right)
\end{aligned}
$$

where $\Delta \bar{h}_{m}\left(t_{D}\right)$ is given by

$$
\Delta \bar{h}_{m}\left(t_{D}\right)=\sum_{t=\left(t_{D}-1\right) N_{T}+1}^{t_{D} N_{T}} \frac{n_{m}(t)}{\sqrt{\beta E_{D}} N_{T} d_{m, P}} .
$$

It is obvious that channel estimation suffers from AWGN, which results in imperfect channel estimation. Because the channel estimation error has direct contribution to decision 
noise (as it will be shown in the following analysis), it must be suppressed as much as possible. The channel estimation error can be modeled as a Gaussian noise uncorrelated for different $t_{D}$. With the slow fading channel assumption, i.e., the fading remains almost constant in recent $N_{D}$ pilot symbols' duration, more accurate channel estimation can be obtained by

$$
\begin{aligned}
\overline{\bar{h}}_{m}\left(t_{D}\right) & =\frac{1}{N_{D}} \sum_{i=\left\lceil-\frac{N_{D}}{2}\right\rceil}^{\left\lceil\frac{N_{D}}{2}\right\rceil-1} \bar{h}_{m}\left(t_{D}-i\right) \\
& =h_{m}+\frac{1}{N_{D}} \sum_{i=\left\lceil-\frac{N_{D}}{2}\right\rceil}^{\left\lceil\frac{N_{D}}{2}\right\rceil-1} \Delta \bar{h}_{m}\left(t_{D}-i\right) \\
& \equiv h_{m}+\Delta \overline{\bar{h}}_{m}
\end{aligned}
$$

where the channel estimation error $\Delta \overline{\bar{h}}_{m}$ is given by

$$
\Delta \overline{\bar{h}}_{m}=\frac{1}{N_{D}} \sum_{i=\left\lceil-\frac{N_{D}}{2}\right\rceil}^{\left\lceil\frac{N_{D}}{2}\right\rceil-1} \Delta \bar{h}_{m}\left(t_{D}-i\right) .
$$

Since $h_{m}$ and $\Delta \bar{h}_{m}\left(\lambda_{D}\right)$ are uncorrelated Gaussian variables with zero mean, channel estimation error $\overline{\bar{h}}_{m}\left(\lambda_{D}\right)$ can also be regarded as a Gaussian variable uncorrelated with $h_{m}$. The conditional mean and variance of $\overline{\bar{h}}_{m}$ are

$$
\mu_{\overline{\bar{h}}_{m} \mid h_{m}}=E\left\{\overline{\bar{h}}_{m} \mid h_{m}\right\}=h_{m}
$$

and

$$
\begin{aligned}
R_{\overline{\bar{h}}_{m} \mid h_{m}} & =\frac{1}{2} E\left\{\left(\overline{\bar{h}}_{m}-E\left\{\overline{\bar{h}}_{m}\right\}\right)\left(\overline{\bar{h}}_{m}-E\left\{\overline{\bar{h}}_{m}\right\}\right)^{*} \mid h_{m}\right\} \\
& =\frac{\sigma^{2}}{\beta E_{D} N_{T} N_{D}}
\end{aligned}
$$

respectively.
Intuitively, from (2), noise power $\left(\sigma^{2}\right)$ estimation can be calculated with time domain despreading output (6) by

$$
\hat{\sigma}^{2}=\frac{1}{2 N_{T} M} \sum_{m=1}^{M}\left|y_{m, 1}\left(t_{D}\right)-\sqrt{\beta E_{D}} N_{T} h_{m} d_{m, P}\right|^{2} .
$$

Then, noise power estimation is unbiased, i.e., $E\left\{\hat{\sigma}^{2}\right\}=\sigma^{2}$. However, since only estimated channel information is available at the receiver, (13) can be approximated as that shown in (14) at the bottom of the page. It can be shown from (14) that $E\left\{\hat{\sigma}^{2}\right\}=$ $\left(N_{D}-1\right) \sigma^{2} / N_{D}$. It means that in case of imperfect channel estimation, the approximated noise power estimation is biased. To maintain unbiased estimation, the estimated noise power is adjusted by

$$
\begin{aligned}
\hat{\sigma}^{2}= & \frac{N_{D}}{2 N_{T} M\left(N_{D}-1\right)} \\
& \times \sum_{m=1}^{M}\left|y_{m, 1}\left(t_{D}\right)-\sqrt{\beta E_{D}} N_{T} \overline{\bar{h}}_{m}\left(t_{D}\right) d_{m, P}\right|^{2} \\
\equiv & \sum_{m=1}^{M}\left|\hat{n}_{m}\left(t_{D}\right)\right|^{2}
\end{aligned}
$$

where $\hat{n}_{m}\left(t_{D}\right)$ is expressed as that shown in (16) at the bottom of the page.

Since $\hat{n}_{m}\left(t_{D}\right)$ is the sum of independent Gaussian variables, it can be approximated as a statistically independent and identically distributed complex Gaussian variable with zero mean and variance of

$$
\sigma_{1}^{2}=\frac{1}{2} E\left\{\left|\hat{n}_{m}\left(t_{D}\right)\right|^{2}\right\}=\frac{\sigma^{2}}{2 M} .
$$

Therefore, noise power estimation is unbiased and can be regarded as a central chi-square-distributed random variable with $2 M$ degrees of freedom.

For simplification, the symbol index $t_{D}$ will be dropped out.

$$
\begin{aligned}
\hat{\sigma}^{2} & \approx \frac{1}{2 N_{T} M} \sum_{m=1}^{M}\left|y_{m, 1}\left(t_{D}\right)-\sqrt{\beta E_{D}} N_{T} \overline{\bar{h}}_{m}\left(t_{D}\right) d_{m, P} C^{(S C)}(m)\right|^{2} \\
& =\frac{1}{2 N_{T} M} \sum_{m=1}^{M}\left|-\frac{1}{N_{D}} \sum_{t_{D}^{\prime}=t_{D}+\left\lceil-\frac{N_{D}}{2}\right\rceil t}^{t_{D}+\left\lceil\frac{N_{D}}{2}\right\rceil-1} \sum_{t=\left(t_{D}^{\prime}-1\right) N_{T}+1}^{t_{D}^{\prime} N_{T}} n_{m}(t)+\sum_{t=\left(t_{D}-1\right) N_{T}+1}^{t_{D} N_{T}} n_{m}(t)\right|^{2}
\end{aligned}
$$

$$
\hat{n}_{m}\left(t_{D}\right)=\frac{-\sum_{t_{D}^{\prime}=t_{D}+\left\lceil-\frac{N_{D}}{2}\right\rceil t=\left(\sum_{D}^{\prime}-1\right) N_{T}+1}^{t_{D}+\left\lceil\frac{N_{D}}{2}\right\rceil-1} \frac{n_{m}(t)}{N_{D}}+\sum_{t=\left(t_{D}-1\right) N_{T}+1}^{t_{D} N_{T}} n_{m}(t)}{\sqrt{\frac{2 N_{T} M\left(N_{D}-1\right)}{N_{D}}}}
$$




\section{Optimum Detector}

The optimum detector is to select the vector $\hat{\mathbf{d}}$ from all possible combinations of the transmitted vectors $\{\mathbf{d}\}$ that maximize the a posteriori probability $p\left(\mathbf{d} \mid \mathbf{Y}_{\hat{k}}\right)$, where $\mathbf{d}=\left[d_{1, \hat{k}-1}, d_{1, \hat{k}-1+\left(N_{T}-1\right)}, \ldots, d_{1, \hat{k}-1+\left(K_{c}-1\right)\left(N_{T}-1\right)}\right]^{T} \quad \mathrm{de}-$ notes the transmitted symbol vector. It is well known that maximizing the a posteriori probability is equivalent to maximizing the likelihood $p\left(\mathbf{Y}_{\hat{k}} \mid \mathbf{d}\right)$ when equiprobable symbols are transmitted.

From (3) and (5), conditioned on $\mathbf{d}, \mathbf{Y}_{\hat{k}}$ is Gaussian with a mean and variance of

$$
\boldsymbol{\mu}_{\mathbf{Y}_{\hat{k}} \mid \mathbf{d}}=E\left\{\mathbf{Y}_{\hat{k}} \mid \mathbf{d}\right\}=\left[\mu_{1}, \mu_{2}, \ldots, \mu_{N_{F}}\right]^{T}
$$

and

$$
\begin{aligned}
R_{\mathbf{Y}_{\hat{k}} \mid \mathbf{d}} & =\frac{1}{2} E\left\{\left(\mathbf{Y}_{\hat{k}}-\boldsymbol{\mu}_{\mathbf{Y}_{\hat{k}} \mid \mathbf{d}}\right)\left(\mathbf{Y}_{\hat{k}}-\boldsymbol{\mu}_{\mathbf{Y}_{\hat{k}} \mid \mathbf{d}}\right)^{H} \mid \mathbf{d}\right\} \\
& =\frac{1}{2} E\left\{\boldsymbol{\eta} \boldsymbol{\eta}^{H}\right\}
\end{aligned}
$$

where $\quad \mu_{m}=\sqrt{E_{D}} N_{T} h_{m} c^{(S C)}(m) \sum_{\hat{k}_{f}=1}^{K_{c}} c_{N_{F}, \hat{k}_{f}}(m)$ $d_{1, \hat{k}-1+\left(\hat{k}_{f}-1\right)\left(N_{T}-1\right)}$ and $\boldsymbol{\eta} \equiv\left[\begin{array}{llll}\eta_{1, \hat{k}} & \eta_{2, \hat{k}} & \ldots & \eta_{N_{F}, \hat{k}}\end{array}\right]^{T}$. Since AWGN components on different subcarriers are uncorrelated, we obtain $R_{\mathbf{Y}_{\hat{k}} \mid \mathbf{d}}=N_{T} \sigma^{2} \mathbf{I}$, where $\mathbf{I}$ is the identity matrix.

Then, the conditional Gaussian variable $\mathbf{Y}_{\hat{k}}$ has the probability density function $p\left(\mathbf{Y}_{\hat{k}} \mid \mathbf{d}\right)$ as

$p\left(\mathbf{Y}_{\hat{k}} \mid \mathbf{d}\right)=\frac{1}{\left(2 \pi N_{T} \sigma^{2}\right)^{N_{F}}} \exp \left(-\frac{\sum_{m=1}^{N_{F}}\left|y_{m, \hat{k}}-\mu_{m}\right|^{2}}{2 N_{T} \sigma^{2}}\right)$.

Maximizing $p\left(\mathbf{Y}_{\hat{k}} \mid \mathbf{d}\right)$ is equivalent to minimizing its negative exponent, i.e.,

$$
\Lambda(\mathbf{d}) \equiv \sum_{m=1}^{N_{F}}\left|y_{m, \hat{k}}-\overline{\bar{\mu}}_{m}\right|^{2}
$$

where $\quad \overline{\bar{\mu}}_{m}=\sqrt{E_{D}} N_{T} \overline{\bar{h}}_{m} c^{(S C)}(m) \sum_{\hat{k}_{f}=1}^{K_{c}} c_{N_{F}, \hat{k}_{f}}(m)$ $d_{1, \hat{k}-1+\left(\hat{k}_{f}-1\right)\left(N_{T}-1\right)}$, i.e., $h_{m}$ is replaced with $\overline{\bar{h}}_{m}$ in $\mu_{m}$ for the fact that only the channel estimation is available at the receiver. Omitting constants in (21), one obtains

$$
\bar{\Lambda}(\mathbf{d})=\sum_{m=1}^{N_{F}}\left|\overline{\bar{\mu}}_{m}\right|^{2}-\sum_{m=1}^{N_{F}} 2 \Re\left\{y_{m, \hat{k}}^{*} \overline{\bar{\mu}}_{m}\right\}
$$

where $\Re\{x\}$ stands for real part of $x$. Therefore, the transmitted symbols are recovered by selecting the $\mathbf{d}$ to minimize $\bar{\Lambda}(\mathbf{d})$.

\section{E. MMSE Detector}

It is well known that the computation complexity of the optimal detector increases exponentially as the number of cochannel signals $K_{c}$ increases. In order to reduce the complexity when $K_{c}$ is large, MMSE is studied.
The MMSE detector is to minimize the mean square error $J_{\hat{k}, \hat{k}_{f}}=E\left\{\left|d_{1, \hat{k}-1+\left(\hat{k}_{f}-1\right)\left(N_{T}-1\right)}-\mathbf{w}_{\hat{k}, \hat{k}_{f}}^{H} \mathbf{Y}_{\hat{k}}\right|^{2} \mid \mathbf{d}\right\}$, where $\mathbf{w}_{\hat{k}, \hat{k}_{f}}$ is the weight vector for the $k$ th code channel. Then, the data decision can be made by

$$
\hat{d}_{1, \hat{k}-1+\left(\hat{k}_{f}-1\right)\left(N_{T}-1\right)}=\operatorname{sgn}\left[\Re\left(\mathbf{w}_{\hat{k}, \hat{k}_{f}}^{H} \mathbf{Y}_{\hat{k}}\right)\right]
$$

where $\operatorname{sgn}(x)=-1$ and 1 for $x<0$ and $x \geq 0$, respectively.

Solving the zero point of the differential of mean square error $J_{\hat{k}, \hat{k}_{f}}$ with respect to $\mathbf{w}_{\hat{k}, \hat{k}_{f}}$, the weighting factor $\mathbf{w}_{\hat{k}, \hat{k}_{f}}$ can be obtained by

$$
\begin{aligned}
\mathbf{w}_{\hat{k}, \hat{k}_{f}}= & E\left\{\mathbf{Y}_{\hat{k}} \mathbf{Y}_{\hat{k}}^{H} \mid \mathbf{d}\right\}^{-1} d_{1, \hat{k}-1+\left(\hat{k}_{f}-1\right)\left(N_{T}-1\right)} E\left\{\mathbf{Y}_{\hat{k}} \mid \mathbf{d}\right\} \\
= & \left(E\left\{\boldsymbol{\mu}_{\mathbf{Y}_{\hat{k}} \mid \mathbf{d}} \boldsymbol{\mu}_{\mathbf{Y}_{\hat{k}} \mid \mathbf{d}}^{H}\right\}+E\left\{\boldsymbol{\eta} \boldsymbol{\eta}^{H}\right\}\right)^{-1} \\
& \times\left[\begin{array}{c}
\sqrt{E_{D}} N_{T} h_{1} c^{(S C)}(1) C_{N_{F}, \hat{k}_{f}}(1) \\
\sqrt{E_{D}} N_{T} h_{2} c^{(S C)}(2) C_{N_{F}, \hat{k}_{f}}(2) \\
\vdots \\
\sqrt{E_{D}} N_{T} h_{N_{F}} c^{(S C)}\left(N_{F}\right) C_{N_{F}, \hat{k}_{f}}\left(N_{F}\right)
\end{array}\right]
\end{aligned}
$$

where the desired signal components and AWGN components are uncorrelated with each other. With the assumption that independent data symbols are transmitted over different code channels and $E\left|\sum_{\hat{k}_{f}^{\prime}=1}^{K_{c}} C_{N_{F}, \hat{k}_{f}^{\prime}} d_{1, \hat{k}-1+\left(\hat{k}_{f}^{\prime}-1\right)\left(N_{T}-1\right)}\right|^{2}=K_{c}$,
one obtains

$$
\begin{aligned}
R_{\boldsymbol{\mu}} & \equiv E\left\{\boldsymbol{\mu}_{\mathbf{Y}_{\hat{k}} \mid \mathbf{d}} \boldsymbol{\mu}_{\mathbf{Y}_{\hat{k}} \mid \mathbf{d}}^{H}\right\} \\
& =E_{D} K_{c} N_{T}^{2}\left[\begin{array}{cccc}
\left|h_{1}\right|^{2} & 0 & \cdots & 0 \\
0 & \left|h_{2}\right|^{2} & \cdots & 0 \\
\vdots & \vdots & \ddots & \vdots \\
0 & 0 & \cdots & \left|h_{N_{F}}\right|^{2}
\end{array}\right]
\end{aligned}
$$

Since at the receiver the real channel information (including noise power and channel fading) is unknown to the detector, estimated noise power $\hat{\sigma}^{2}$ and channel fading $\overline{\bar{h}}_{m}$ are used to construct the weight vector as

$$
\mathbf{w}_{\hat{k}, \hat{k}_{f}}=\left[w_{\hat{k}, \hat{k}_{f}}(1), w_{\hat{k}, \hat{k}_{f}}(2), \ldots, w_{\hat{k}, \hat{k}_{f}}\left(N_{F}\right)\right]^{T}
$$

where $\quad w_{\hat{k}, \hat{k}_{f}}(m)=\left(\sqrt{E_{D}} \overline{\bar{h}}_{m} c^{(S C)}(m) C_{N_{F}, \hat{k}_{f}}(m)\right) /$ $\left(E_{D} N_{T} K_{c}\left|\overline{\bar{h}}_{m}\right|^{2}+2 \hat{\sigma}^{2}\right)$.

Substituting (26) into (23), the data decision for the symbol on the $k$ th code channel can be made.

\section{BER PERFORMANCE ANALYSIS}

\section{A. Performance of Optimum Detector}

The upper union bound on the BER performance of the optimum detector for the $k$ th code channel $p_{b, k}$ is given. Letting $\mathbf{d}_{l}$ denote the transmitted vector, an error occurs in the receiver when the detector finds a vector $\mathbf{d}_{q}$ that satisfies $\bar{\Lambda}\left(\mathbf{d}_{q}\right)<\bar{\Lambda}\left(\mathbf{d}_{l}\right)$ with $\mathbf{d}_{l} \neq \mathbf{d}_{q}$ and with probability $p\left(\bar{\Lambda}\left(\mathbf{d}_{q}\right)<\bar{\Lambda}\left(\mathbf{d}_{l}\right)\right)$ of this pairwise. Then, the union bound can 
be obtained by summing $p\left(\bar{\Lambda}\left(\mathbf{d}_{q}\right)<\bar{\Lambda}\left(\mathbf{d}_{l}\right)\right)$ over all vectors (denoted as $\overline{\left\{\mathbf{d}_{l}\right\}}$ ) that differ from $\mathbf{d}_{l}$ in their $k$ th position and then averaging over all possible transmitted vector $\mathbf{d}_{l}$, which is expressed as

$$
p_{b, k}<\frac{1}{2^{K_{c}}} \sum_{\mathbf{d}_{l}} \sum_{\mathbf{d}_{q} \in \overline{\left\{\mathbf{d}_{l}\right\}}} p\left(\bar{\Lambda}\left(\mathbf{d}_{q}\right)<\bar{\Lambda}\left(\mathbf{d}_{l}\right) \mid \mathbf{d}_{l}\right) .
$$

Considering the symmetric property of the transmitted vector, $p_{b, k}$ can be written as

$$
p_{b, k}<\sum_{\mathbf{d}_{q} \in \overline{\left\{\mathbf{d}_{l}\right\}}} p\left(\bar{\Lambda}\left(\mathbf{d}_{q}\right)<\bar{\Lambda}\left(\mathbf{d}_{l}\right) \mid \mathbf{d}_{l}\right)=\sum_{\mathbf{d}_{q} \in \overline{\left\{\mathbf{d}_{l}\right\}}} p\left(D_{l, q}<0 \mid \mathbf{d}_{l}\right)
$$

where $D_{l, q}=\bar{\Lambda}\left(\mathbf{d}_{q}\right)-\bar{\Lambda}\left(\mathbf{d}_{l}\right)$.

Defining the equivalent symbol transmitted on the $m$ th subcarrier $\bar{d}^{(m)}$ as

$$
\bar{d}^{(m)}=\sum_{\hat{k}_{f}^{\prime}=1}^{K_{c}} C_{N_{F}, \hat{k}_{f}^{\prime}}(m) d_{1, \hat{k}-1+\left(\hat{k}_{f}^{\prime}-1\right)\left(N_{T}-1\right)}
$$

and the vector $\mathbf{z}_{m}=\left[y_{m, \hat{k}} \sqrt{E_{D}} N_{T} \overline{\bar{h}}_{m} c^{(S C)}(m)\right]^{T}$, we obtain $D_{l, q}=\bar{\Lambda}\left(\mathbf{d}_{q}\right)-\bar{\Lambda}\left(\mathbf{d}_{l}\right)=\sum_{m=1}^{N_{F}} \mathbf{z}_{m}^{H} \mathbf{F}_{l, q}^{(m)} \mathbf{z}_{m}$. Then, $p\left(D_{l, q}<\right.$ $\left.0 \mid \mathbf{d}_{l}\right)$ is calculated by

$$
p\left(D_{l, q}<0 \mid \mathbf{d}_{l}\right)=\sum_{s \in \text { right poles of } \Phi_{D_{l, q}}(s)} \text { residue }\left\{\Phi_{D_{l, q}}(s)\right\}
$$

where $\Phi_{D_{l, q}}(s)$ is the moment generating function (MGF) of $D_{l, q}$ given by

$$
\Phi_{D_{l, q}}(s)=\prod_{m=1}^{N_{F}} \frac{1}{\operatorname{det}\left(\mathbf{I}+2 s \mathbf{R}^{(m)} \mathbf{F}_{l, q}^{(m)}\right)}
$$

where the Hermitian matrix $\mathbf{F}_{l, q}^{(m)}$ is expressed as that shown in (32) at the bottom of the page and $\mathbf{R}^{(m)}=(1 / 2) E\left\{\mathbf{z}_{m} \mathbf{z}_{m}^{H} \mid \mathbf{d}_{l}\right\}$ is expressed as

$$
\mathbf{R}^{(m)}=\left[\begin{array}{cc}
\left(E_{D} N_{T}^{2}\left|\bar{d}_{l}^{(m)}\right|^{2}+N_{T} \sigma^{2}\right) & E_{D} N_{T}^{2}\left(\bar{d}_{l}^{(m)}\right)^{T} \\
E_{D} N_{T}^{2}\left(\bar{d}_{l}^{(m)}\right)^{*} & E_{D} N_{T}^{2}\left(1+\frac{\sigma^{2}}{N_{D} \beta E_{D} N_{T}}\right)
\end{array}\right] .
$$

Now we study two different cases to derive $1 / \operatorname{det}(\mathbf{I}+$ $\left.2 s \mathbf{R}^{(m)} \mathbf{F}_{l, q}^{(m)}\right)$ for $m=1,2, \ldots, N_{F}$. When $\bar{d}_{q}^{(m)} \neq \bar{d}_{l}^{(m)}$, we find that $\left|\mathbf{F}_{l, q}^{(m)}\right|$ and $\left|\mathbf{R}^{(m)}\right|$ are nonzero and the matrix $2 \mathbf{R}^{(m)} \mathbf{F}_{l, q}^{(m)}$ is a full rank, i.e., the rank of $2 \mathbf{R}^{(m)} \mathbf{F}_{l, q}^{(m)}$ is two. Thus, $2 \mathbf{R}^{(m)} \mathbf{F}_{l, q}^{(m)}$ has only two nonzero eigenvalues, i.e.,

$$
\frac{1}{\operatorname{det}\left(\mathbf{I}+2 s \mathbf{R}^{(m)} \mathbf{F}_{l, q}^{(m)}\right)}=\frac{p_{l, q, 1}^{(m)} p_{l, q, 2}^{(m)}}{\left(s-p_{l, q, 1}^{(m)}\right)\left(s-p_{l, q, 2}^{(m)}\right)}
$$

where $-1 / p_{l, q, 1}^{(m)}$ and $-1 / p_{l, q, 2}^{(m)}$ are the two nonzero eigenvalues of $2 \mathbf{R}^{(m)} \mathbf{F}_{l, q}^{(m)}$. When $\bar{d}_{q}^{(m)}=\bar{d}_{l}^{(m)}, \mathbf{F}_{l, q}^{(m)}=\mathbf{0}$, and $\mathbf{I}+2 s \mathbf{R}^{(m)} \mathbf{F}_{l, q}^{(m)}=\mathbf{I}, \Phi_{D_{l, q}}(s)=1$.

Finally, using the Gauss-Chebychev quadrature method [8], (30) can be calculated and the overall BER union bound (or the BER bound for one code channel) is

$$
p_{b}=E\left\{p_{b, k}\right\} .
$$

\section{B. Performance of the MMSE Detector}

For the MMSE detector, the BER for the $k$ th code channel $p_{b, k}$ is $p_{b, k}=p\left(\hat{d}_{1, k}=-1 \mid d_{1, k}=1\right)$, where $\hat{d}_{1, k}$ is obtained from (23). Conditioned on channel estimation $\left\{\bar{h}_{m}\right\}$, noise power estimation $\hat{\sigma}^{2}$, and the transmitted symbol vector $\mathbf{d}$, $w_{\hat{k}, \hat{k}_{f}}^{*}(m) y_{m, \hat{k}}$ is a Gaussian variable with mean

$$
\begin{aligned}
E\left\{w_{\hat{k}, \hat{k}_{f}}^{*}(m) y_{m, \hat{k}} \mid\left\{\overline{\bar{h}}_{m}\right\}, \hat{\sigma}^{2}, \mathbf{d}\right\} & \\
& =\frac{E_{D} N_{T}\left|\overline{\bar{h}}_{m}\right|^{2} C_{N_{F}, \hat{k}_{f}}(m) \bar{d}^{(m)}}{E_{D} N_{T} K_{c}\left|\overline{\bar{h}}_{m}\right|^{2}+2 \hat{\sigma}^{2}}
\end{aligned}
$$

and variance expressed as that shown in (37) at the bottom of the page.

$$
\begin{aligned}
\mathbf{F}_{l, q}^{(m)} & =\left[\begin{array}{c}
1 \\
-\bar{d}_{q}^{(m)}
\end{array}\right]^{*}\left[\begin{array}{ll}
1 & -\bar{d}_{q}^{(m)}
\end{array}\right]-\left[\begin{array}{c}
1 \\
-\bar{d}_{l}^{(m)}
\end{array}\right]^{*}\left[\begin{array}{cc}
1 & -\bar{d}_{l}^{(m)}
\end{array}\right] \\
& =\left[\begin{array}{c}
0 \\
-\left(\bar{d}_{q}^{(m)}-\bar{d}_{l}^{(m)}\right)^{T} \\
-\left(\bar{d}_{q}^{(m)}-\bar{d}_{l}^{(m)}\right)^{*}\left(\bar{d}_{q}^{(m)}\right)^{*}\left(\bar{d}_{q}^{(m)}\right)^{T}-\left(\bar{d}_{l}^{(m)}\right)^{*}\left(\bar{d}_{l}^{(m)}\right)^{T}
\end{array}\right]
\end{aligned}
$$

$$
\operatorname{Var}\left\{w_{\hat{k}, \hat{k}_{f}}^{*}(m) y_{m, \hat{k}} \mid\left\{\overline{\bar{h}}_{m}\right\}, \hat{\sigma}^{2}, \mathbf{d}\right\}=\frac{E_{D}\left|\overline{\bar{h}}_{m}\right|^{2} E\left\{\left|-\sqrt{E_{D}} N_{T} \Delta \overline{\bar{h}}_{m} C_{N_{F}, \hat{k}_{f}}(m) \bar{d}^{(m)}+\left(c^{(S C)}(m) C_{N_{F}, \hat{k}_{f}}(m)\right)^{*} \eta_{m, \hat{k}}\right|^{2}\right\}}{2\left(E_{D} N_{T} K_{c}\left|\overline{\bar{h}}_{m}\right|^{2}+2 \hat{\sigma}^{2}\right)^{2}}
$$


With independent AWGN noise on different subcarriers, the conditional variance is

$$
\begin{aligned}
\operatorname{Var}\left\{w_{\hat{k}, \hat{k}_{f}}^{*}(m) y_{m, \hat{k}} \mid\left\{\overline{\bar{h}}_{m}\right\}, \hat{\sigma}^{2}, \mathbf{d}\right\} \\
=\frac{E_{D} N_{T}\left|\overline{\bar{h}}_{m}\right|^{2}\left(E_{D} N_{T}\left|\bar{d}^{(m)}\right|^{2} R_{\overline{\bar{h}} \mid h}+\sigma^{2}\right)}{\left(E_{D} N_{T} K_{c}\left|\overline{\bar{h}}_{m}\right|^{2}+2 \hat{\sigma}^{2}\right)^{2}} .
\end{aligned}
$$

Then, the output signal to noise and interference ratio (SINR) is

$$
\gamma_{o}=\frac{\left(\Re\left\{\sum_{m=1}^{N_{F}} E\left\{w_{\hat{k}, \hat{k}_{f}}^{*}(m) y_{m, \hat{k}} \mid\left\{\overline{\bar{h}}_{m\}}, \hat{\sigma}^{2}, \mathbf{d}\right\}\right\}\right)^{2}\right.}{2} .
$$

So, the conditional BER is given by

$$
p\left(\hat{d}_{1, k}=-1 \mid d_{1, k}=1,\left\{\overline{\bar{h}}_{m}\right\}, \hat{\sigma}^{2}, \mathbf{d}\right)=Q\left(\sqrt{2 \gamma_{o}}\right)
$$

where $Q(\bullet)$ is the $Q$-function defined as $Q(x)=$ $(1 / \sqrt{2 \pi}) \int_{x}^{\infty} e^{-t^{2} / 2} \mathrm{~d} t$.

Calculating the expectation over the joint ensemble of channel, noise power estimates, and all possible ds with $d_{1, k}=1$, we can get the average BER for the $k$ th code channel

$$
\begin{aligned}
p_{b, k} & =p\left(\hat{d}_{1, k}=-1 \mid d_{1, k}=1\right) \\
& =E_{\left\{\overline{\bar{h}}_{m}\right\}, \hat{\sigma}^{2}, \mathbf{d}}\left\{p\left(\hat{d}_{1, k}=-1 \mid d_{1, k}=1,\left\{\overline{\bar{h}}_{m}\right\}, \hat{\sigma}^{2}, \mathbf{d}\right)\right\} .
\end{aligned}
$$

After calculating (41) through the numerical method, the analytical probability of bit error for the overall system is obtained by (35).

\section{Numerical Results}

In this section, some representative numerical results are presented. The effect of different system parameters such as spreading factors, noise, and channel parameter estimations on the BER performance is investigated by numerical evaluation. The number of subcarriers $M$ is 768 and the OFCDM symbol period (plus guard interval) is $8 \mu$ s. Unless noted otherwise, the recent received 48 OFCDM symbols are collected for channel estimation and the chip energy ratio of pilot channel to one code data channel $\beta$ is $15 \mathrm{~dB}$. The ISI is efficiently suppressed by proper setting of the guard interval. The average SNR for the transmitted data is $10 \mathrm{~dB}$, which is defined as $\mathrm{SNR}=N E_{D} / \sigma_{n}^{2}$.

First, we investigate the BER performance with different detectors by means of simulation and analytical methods. Fig. 5 shows the BER performance versus SNR when the spreading factor is $N=16 \times 16$ and the number of code channels is 60 . It can be seen that the optimal detector significantly outperforms the MMSE detector, especially in large SNRs. This is mainly because the optimal detector can make a simultaneous decision

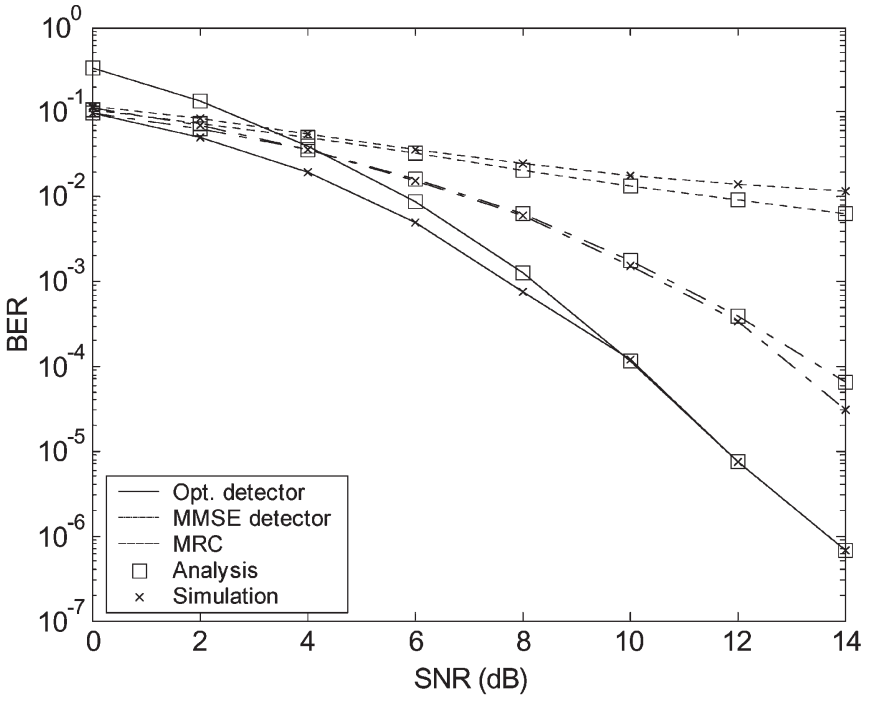

Fig. 5. Analytical and simulation BER versus SNR for different detectors.

on data transmitted over all $K_{c}$ code channels, which counts the effect of all the transmitted code channels. Although, with the additive Gaussian assumption for MCI, the MMSE detector can suppress the interference from other code channels, it cannot make full use of the information from other code channels, which results in significant performance degradation, compared to the optimal detector. However, the MMSE receiver outperforms the maximum ratio combining (MRC) receiver. In addition to the AWGN component, $\mathrm{MCI}$ is another important additive interference. However, MRC cannot combat MCI, whereas the MMSE detector takes into account the effect of both MCI and the presence of AWGN. Thus, the MMSE detector can provide significant performance improvement compared to the MRC detector.

Moreover, simulation results are plotted to verify the accuracy of the analytical results. During the simulations, the guard interval is set at $25 \%$ of an OFCDM symbol period as configured in [1], i.e., $2 \mu \mathrm{s}$, the Doppler frequency is set at $20 \mathrm{~Hz}$, and a 24-path Rayleigh fading channel is assumed. It can be seen that analytical results are very close to simulation ones for all receivers, except for the optimal detector in the case of a small SNR. Therefore, when $K_{c}$ is large, the MMSE detector is employed due to its low computation complexity.

Fig. 6 shows the BER performance for a given channel load $K / N$ and for various values $(4,8,16)$ of the frequency domain spreading factor $N_{F}$ when the time domain spreading factor is 16 . Note that for a given $K / N, K$ increases linearly as $N$ increases. It can be seen that for the MMSE detector, the frequency domain spreading factor does not affect system performance much. A large $N_{F}$ results in a large $K$ so that more MCI is caused. Although a large $N_{F}$ provides high frequency diversity for MMSE, the frequency diversity gain is eliminated by the corresponding large MCI. On the other hand, for the optimum detector, a larger $N_{F}$ can achieve better performance due to the higher frequency diversity gain. This is because the optimum detector demodulates the transmitted symbols simultaneously so that the interference from other code channels can be fully exploited. Therefore, for a given channel 


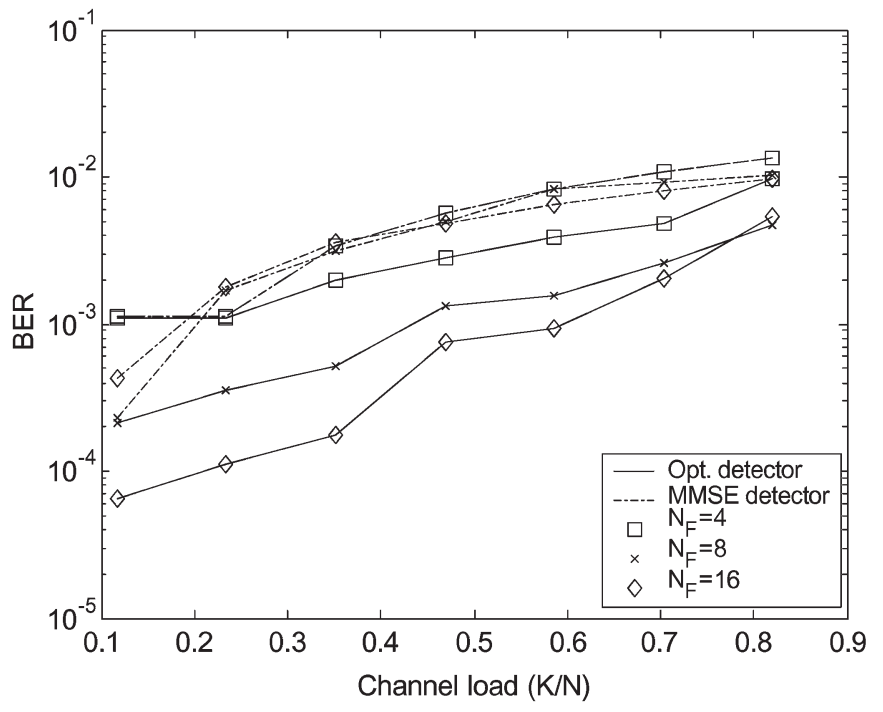

Fig. 6. BER versus channel load $(K / N)$ with different frequency domain spreading factor for a given time domain spreading factor $\left(N_{T}=16\right)$.

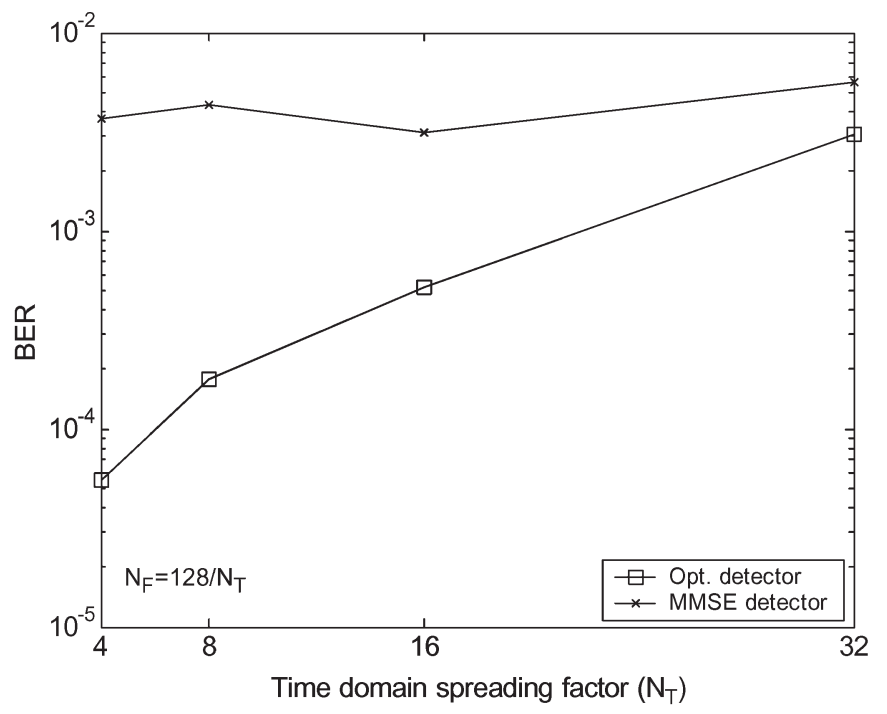

Fig. 7. BER versus time domain spreading factor $\left(N_{T}\right)$ for a given overall spreading factor (channel load $K / N=45 / 128$ ).

load, a higher frequency domain spreading factor is preferred for optimum detector. But a large $N_{F}$ results in a great number of code channels that will make the optimum detection more complicated. Thus, to make the optimum detector practical, $N_{F}$ should be less than or equal to 16 . Furthermore, compared to the optimum detector, it can be found that MMSE is not sensitive to $N_{F}$.

Fig. 7 shows the BER performance with variable time and frequency domain spreading factors $\left(N_{F}=N / N_{T}\right)$ for a fixed channel load $(K / N=0.352)$ and a fixed overall spreading factor $(N=128)$. It can be seen that the BER performance of MMSE is flat for different values of $N_{T}$. When $N_{T}$ increases, $N_{F}$ decreases, so that there are fewer code channels with the same time domain spreading code. Thus, less MCI is caused. That is, a small $N_{F}$ causes less MCI and smaller frequency diversity gain. Therefore, the BER performance of MMSE is almost independent of $N_{F}$ for a given $N$ and $K / N$. As for the

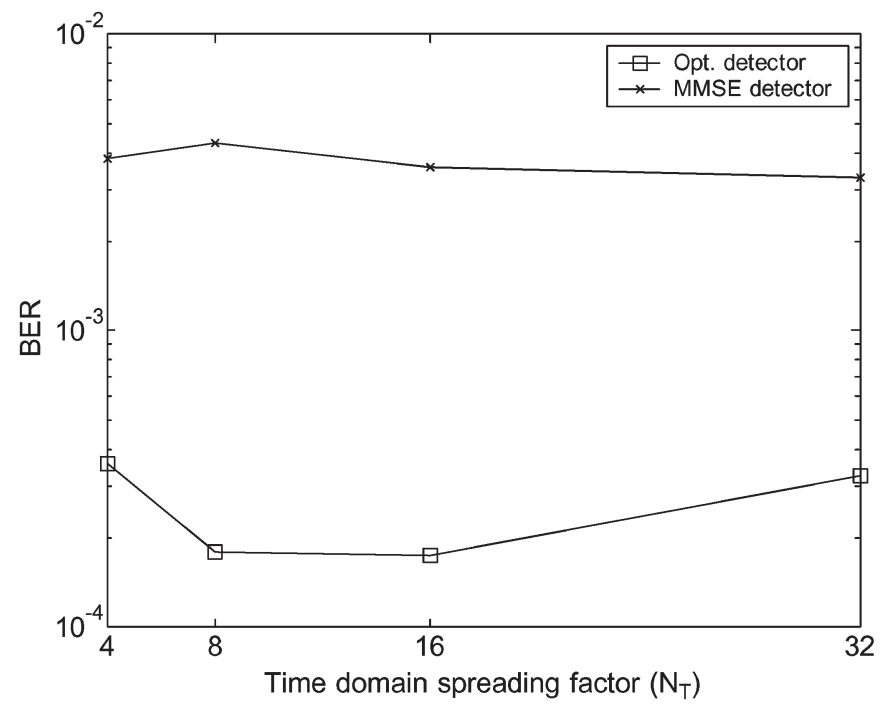

Fig. 8. BER performance versus time domain spreading factor $\left(N_{T}\right)$ for a given time domain spreading factor $\left(N_{F}=16\right)$ and channel load $(K / N=$ $45 / 128)$.

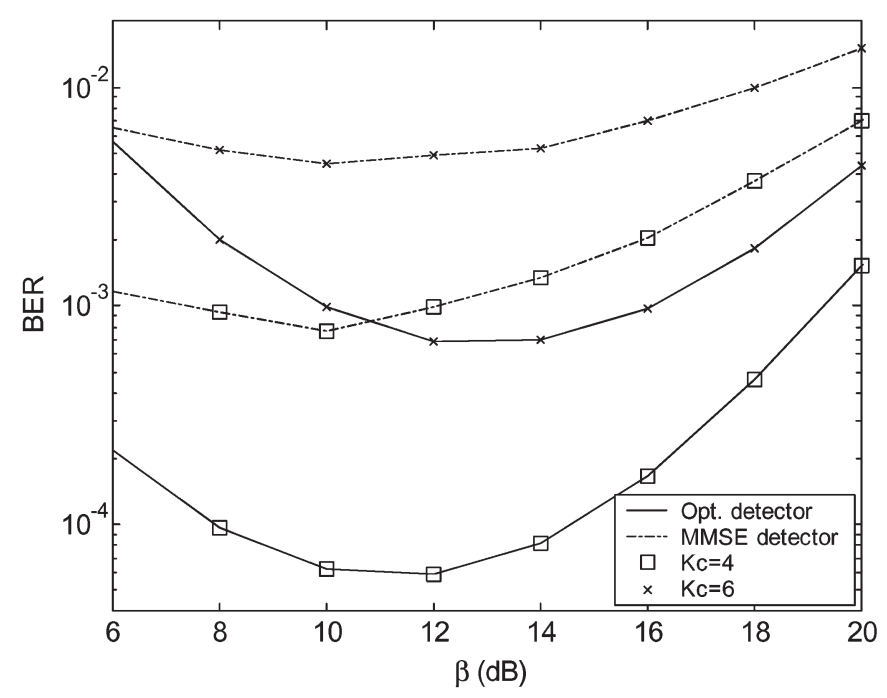

Fig. 9. BER performance with different channel estimation quality $(N=$ $16 \times 16)$.

optimum detector, performance degrades with the increase of $N_{T}$. This is because the performance of the optimum detector is mainly affected by the frequency diversity gain. In general, for a fixed overall spreading factor, a higher frequency domain spreading factor achieves a higher frequency diversity gain by employing an optimum detector.

In Fig. 8, BER performance is shown as a function of $N_{T}$ for a given $N_{F}$ and $K / N$. It can be seen that the performance of both detectors is flat due to the fact that the MCI is almost the same for different values of $N_{T}$ and that frequency diversity gain is given due to a given $N_{F}$.

In Fig. 9, the effect of channel estimation quality on system performance is investigated when the overall transmitted power is fixed, i.e., $\left(K_{c}\left(N_{T}-1\right)+\beta\right) E_{D}$ is fixed. For fair comparison, the variance of AWGN noise is fixed for different $\beta$, so the overall signal to noise power ratio is the same for all $\beta$. In general, channel estimation can be improved by increasing 


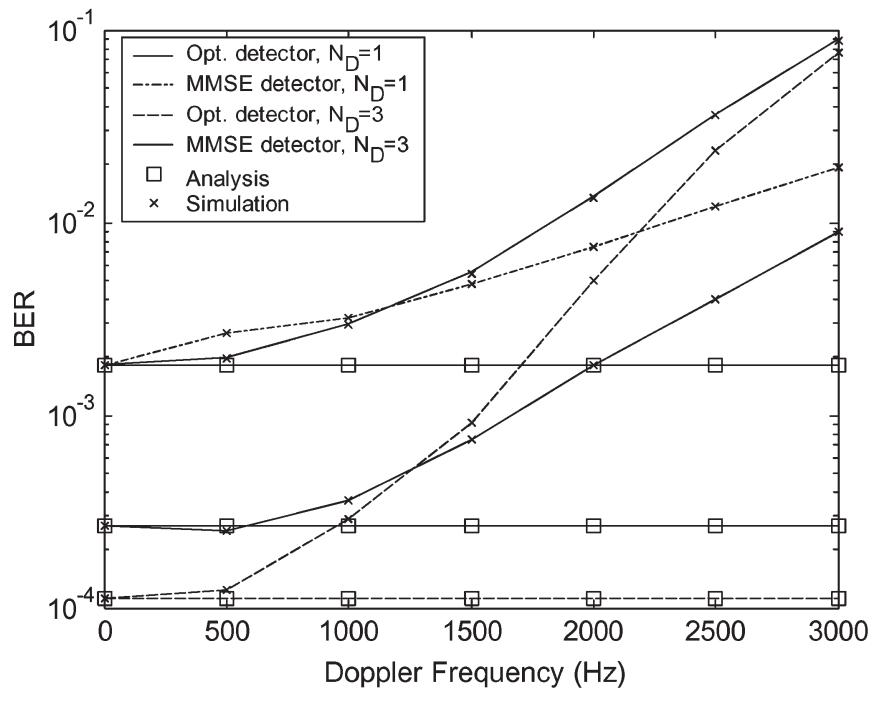

Fig. 10. BER performance versus Doppler frequency with different channel estimation quality and detectors $(N=16 \times 16)$.

the power of the pilot channel. It can be seen that first when $\beta$ increases, the performance is improved dramatically due to more accurate channel estimation. However, since the overall transmitted power is fixed, a larger $\beta$ results in a smaller received SNR for each code data channel due to the decrease of $E_{D}$. So when $\beta$ increases beyond a certain value, the benefit from more accurate channel estimation becomes slight, whereas the received SNR for each code data channel is dominant to system performance; consequently, as shown in the figure, performance degrades with the increase of $\beta$ after it reaches the optimum value $(10 \mathrm{~dB} \sim 14 \mathrm{~dB}$ for MMSE detector with different $K_{c}$ ), i.e., the pilot channel occupies about $15 \%$ of the overall transmitted power. As to optimum detector, a similar scenario can be seen. But the optimum detector jointly detects symbols transmitted on all code channels, then calculation of metric for different symbol combinations is sensitive to channel fading estimation, it requires more accurate channel estimation. Therefore, the optimum value for the optimum detector is greater than that for the MMSE detector under same system conditions. In summary, to achieve better system performance, a tradeoff should be made between the channel estimation quality and the received SNR for code channels.

Finally, the effect of Doppler frequency on system performance is simulated. Simulation conditions are the same as those for Fig. 5, except for the Doppler frequency. In Fig. 10, simulation and analytical results are shown with $N_{D}=1$ and $N_{D}=3$, respectively. Although by (15), noise power estimation cannot be achieved for $N_{D}=1$, to simplify the investigation of MCI and channel fading estimations that is seriously affected by the Doppler frequency, we assume that noise power is perfectly estimated by other advanced algorithms for both cases in this figure. It can be seen that in slow fading cases, i.e., Doppler frequency is less than $500 \mathrm{~Hz}$, analytical results are close to simulation results because the assumption of slow fading is satisfied, so that the provided analytical method can predict system performance well. However, when Doppler frequency increases beyond $500 \mathrm{~Hz}$, the code channels assigned with different time domain spreading codes are no longer orthogonal to each other due to the time varying fading on different chips, which introduces more MCI in time domain. As expected, simulation results show that BER performance degrades dramatically with the increase of Doppler frequency. Moreover, the detectors with $N_{D}=3$ outperform those with $N_{D}=1$ when the Doppler frequency is less than $500 \mathrm{~Hz}$, but as the Doppler frequency increases, the BER performance degrades more for $N_{D}=3$ than that for $N_{D}=1$. This is because although the channel estimation in (9) can suppress the AWGN noise more with a larger $N_{D}$, the desired component is distorted more in fast fading. Therefore, a tradeoff should be made between the noise and Doppler fading effect, and an optimum $N_{D}$ should exist, which is associated with Doppler shift.

\section{CONCLUSion}

In this paper, we have studied the performance of variable spreading factor orthogonal frequency and VSF-OFCDM systems for high data rate service. As the OFCDM signal seriously suffers from MCI in frequency-selective fading channels, optimum and MMSE detectors are compared in this paper. We have derived an analytical BER performance for both detectors considering imperfect channel estimation over slow frequencyselective fading channels. The main conclusions of this paper can be summarized as follows.

1) The MMSE detector is more robust to different system parameters (such as time domain spreading factor, frequency domain spreading factor, etc.) than the optimal detector. Although the optimum detector outperforms the MMSE detector in most cases, it is too complicated, especially in the case of a large number of code channels.

2) Pilot channel power should be determined by making a tradeoff between the channel estimation quality and the received SNR for each code data channel.

3) The system performance of both detectors is significantly affected by Doppler frequency shift, especially when the Doppler shift is larger than $500 \mathrm{~Hz}$.

\section{ACKNOWLEDGMENT}

The authors would like to thank Dr. K. Higuchi, Dr. H. Atarashi, and Dr. N. Maeda of NTT DoCoMo for their helpful discussion.

\section{REFERENCES}

[1] N. Maeda, H. Atarashi, S. Abeta, and M. Sawahashi, "Pilot channel assisted MMSE combining in forward link for broadband OFCDM packet wireless access," IEICE Trans. Fundam. Electron. Commun. Comput., Sci., vol. E85-A, no. 7, pp. 1635-1646, Jul. 2002.

[2] N. Maeda, Y. Kishiyama, H. Atarashi, and M. Sawahashi, "Variable spreading factor-OFCDM with two dimensional spreading that prioritizes time domain spreading for forward link broadband wireless access," in Proc. IEEE Vehicular Technology Conf. (VTC), Orlando, FL, 2003, pp. 127-132.

[3] J. R. Foerster and L. B. Milstein, "Coded modulation for a coherent DS-CDMA system employing an MMSE receiver in a fading channel," IEEE Trans. Commun., vol. 48, no. 11, pp. 1909-1918, Nov. 2000.

[4] H. V. Poor and S. Verdu, "Probability of error in MMSE multiuser detection," IEEE Trans. Inf. Theory, vol. 43, no. 3, pp. 858-871, May 1997.

[5] M. D'Anna and A. H. Aghvami, "Performance of optimum and suboptimum combining at the antenna array of a W-CDMA system," IEEE J. Sel. Areas Commun., vol. 17, no. 12, pp. 2123-2137, Dec. 1999. 
[6] S. J. Grant and J. K. Cavers, "Performance enhancement through joint detection of cochannel signals using diversity arrays," IEEE Trans. Commun., vol. 46, no. 8, pp. 1038-1049, Aug. 1998.

[7] Y. Q. Zhou, J. Wang, and M. Sawahashi, "Downlink transmission of broadband OFCDM systems-Part I: Hybrid detection," IEEE Trans. Commun., vol. 53, pp. 718-729, Apr. 2005.

[8] S. Benedetto and E. Biglieri, Principles of Digital Transmission With Wireless Applications. New York: Kluwer, 1999.

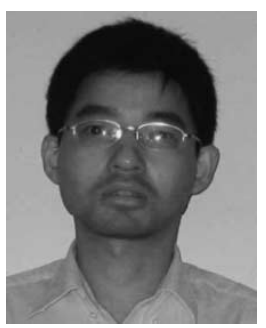

Bin Xia received the B.Eng. degree in electrical engineering and the M.Eng. degree in information and communication engineering from the University of Science and Technology of China, Hefei, China, in 1997 and 2000, respectively, and the Ph.D. degree in electrical engineering from the University of Hong Kong, Hong Kong, in 2004.

From 1995 to 2000, he was with the Personal Communication and Spread Spectrum Laboratory, University of Science and Technology of China, as a Research Engineer involved in the development of code division multiple access (CDMA) communication systems based on IS-95 and Universal Mobile Telecommunications System (UMTS) standards. From 1999 to 2001, he was with UTStarcom Inc., working on wideband CDMA (WCDMA) systems. He is currently a System Engineer at the Alcatel Shanghai Bell Company, Ltd., Shanghai, China. His research interests are in the areas of broadband wireless access technologies, wireless networking, and very large scale integration (VLSI) implementation of wireless transceivers.

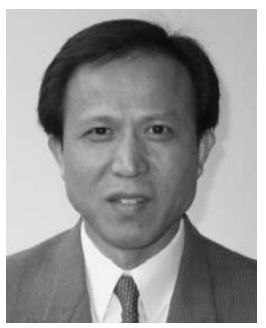

Jiangzhou Wang (M'91-SM'94) received the B.S. and M.S. degrees from Xidian University, Xian, China, in 1983 and 1985, respectively, and the Ph.D. degree (with Greatest Distinction) from the University of Ghent, Gent, Belgium, in 1990, all in electrical engineering.

From 1990 to 1992, he was a Postdoctoral Fellow at the University of California, San Diego, where he worked on the research and development of cellular code division multiple access (CDMA) systems. From 1992 to 1995, he was a Senior System Engineer at Rockwell International Corporation, Newport Beach, CA, where he worked on the development and system design of wireless communications. Since 1995, he has been with the University of Hong Kong, Hong Kong, where he is currently a Professor and the Coordinator of the Telecommunications Group. He has held a Visiting Professor position at NTT DoCoMo, Japan. He has published over 100 papers, including more than 30 IEEE TRANSACTIONS/JOURNALS papers in the areas of wireless mobile and spread spectrum communications. He has written/edited two books entitled Broadband Wireless Communications (Kluwer, 2001) and Advances in $3 G$ Enhanced Technologies for Wireless Communications (Artech House, 2002), respectively. He holds one U.S. patent in the Global System for Mobile Communications (GSM) system and is listed in Who's Who in the World.

Dr. Wang is the Editor for the IEEE TRANSACTIONS ON COMMUNICATIONS and a Guest Editor for the IEEE Journal ON SElEcted AREAS IN COMMUNICATIONS (Wideband CDMA, 8/2000 and 1/2001, and Advances in Multicarrier CDMA, 2006). He was the Technical Chairman of the IEEE Workshop in 3G Mobile Communications 2000.

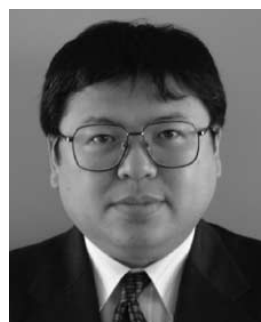

Mamoru Sawahashi (M'88) received the B.S. and M.S. degrees from Tokyo University, Tokyo, Japan, in 1983 and 1985, respectively, and the Dr.Eng. degree from the Nara Institute of Technology, Japan, in 1998.

In 1985, he joined NTT Electrical Communications Laboratories, and in 1992 transferred to NTT Mobile Communications Network, Inc. (now NTT DoCoMo Inc.), Kanagawa, Japan. Since joining NTT, he has been engaged in the research of modulation/demodulation techniques for mobile radio, and research and development of wireless access technologies for wideband code division multiple access (WCDMA) mobile radio and broad-band wireless packet access technologies for beyond IMT-2000. He is currently the Director of the IP Radio Network Development Department of NTT DoCoMo Inc.

Dr. Sawahashi is the Editor for the IEEE TRANSACTIONS ON WIRELESS COMMUNICATIONS and a Guest Editor for the IEEE JOURNAL ON SELECTED AREAS IN COMMUNICATIONS. 